\title{
Palliative care in fetal medicine
}

As our Journal has its main scope in maternal and child health, we understand that the following considerations are pertinent:

In general, palliative care is related to the adult population. The modern hospice movement in the 1970s, created by Cicely Saunders, represents an essential milestone in the construction of what is currently considered as palliative care. Cicely, a social worker, nurse, and physician, defended that pain relief and symptom control are essential in humanized care, which led her to found St. Christopher's Hospice, in London, a reference in research, teaching, and assistance in palliative care. 1

In 1990, the World Health Organization (WHO) defined palliative care, updated in 2002, as being "care provided by a multidisciplinary team that aim to improve the quality of life of patients and their families to face life-threatening illness, through prevention and relief of suffering, as well as early identification, impeccable assessment, and treatment of pain and other physical, social, psychological, and spiritual symptoms".2

Pregnancy is a period of intense physical and psychological changes. A pregnant woman and her family create the most varied expectations, among them is the child's health. Fetal medicine is a specialty constantly in advance with increasingly earlier diagnostic possibilities, using ultrasound and invasive tests, besides intrauterine therapies. Therefore, fetal malformations, including severe or lethal ones, can be detected early in prenatal care.

In countries where abortion is provided by law, when faced with a diagnosis of fetal malformation, families can choose to terminate the pregnancy or have prenatal follow-up. However, there is a paucity of data in literature on family management who choose to keep the pregnancy in case of severe and/or lethal congenital anomaly. The tendency of many obstetricians is to suggest the termination of the pregnancy. The other option offered is to "do nothing." But, it is necessary to understand that families will deal with grief regardless to the outcome of the pregnancy, and palliative care is a way to support families no matter what the option was. ${ }^{3}$ In Brazil, abortion is provided by law, in case of rape, maternal life risk, and, in the context of fetal anomalies, only anencephaly. In cases of other serious or lethal malformations, or those who choose to continue the pregnancy, prenatal should follow as usual.

Breaking the news of a severe or lethal malformation to a mother is an extremely delicate and difficult task. Since the diagnosis to the outpatient follow-up and childbirth, family care can be challenging. Fear, insecurity, and guilt are frequent feelings reported by patients. Some pregnant women remain themselves in denial for a long period of time about their diagnosis. Thus, talking about perinatal palliative care during prenatal care is necessary, although there still seems to be a blockage for many professionals. In the 1980 s there were already studies aiming to apply palliative care in neonatal intensive care units. In 1997, in the United States, there was the first discussion about this type of care in fetuses with lethal anomalies detected during pregnancy. In Brazil, until 2017, there was no prenatal model of palliative care, although it was implemented that year at the Universidade de São Paulo. 4

How would the approach on adopting palliative care at birth be made, depending on the diagnosis, prognosis, and the decisions of the family involved. In cases of anomalies known to be lethal, providing intensive care may be considered irresponsible, and exclusive palliative care would be suggested. The fetal anomalies considered lethal are varied, such as anencephaly, alobar holoprosencephaly, bilateral renal agenesis, bilateral multicystic kidneys, trisomies of chromosomes 13 or 18, among others. 5 It is important to emphasize that a diagnosis of lethal fetal malformation does not necessarily imply immediate death, but intrauterine may occur in the 
peripartum period, at neonatal, or even within months. ${ }^{4}$

The prenatal consultation is the time when the patient usually brings her questions about the diagnostic findings. Establishing a care plan during pregnancy, respecting and supporting the pregnant woman's decisions, with clear information and in accessible language, are essential points during the follow-up. One should pay attention to verbal and non-verbal language, choose a quiet place to communicate about the diagnosis, avoid excessive technical terms, validate the family members' feelings, make pauses and check their understanding, and know how to listen. ${ }^{6}$ It should be understood that it may be necessary to repeat the information in subsequent consultations because sometimes, faced with a difficult diagnosis, patients may not assimilate all the information in a first contact. Obstetricians and pediatricians, nurses, and psychologists, among other team members, can be included in the line of care. It is emphasized that palliative care can be offered early when faced with a severe disease diagnosis, since its objective is to improve the quality of life of patients, even if curative treatment is proposed. 4

Many health professionals have difficulty talking about palliative care, especially in the perinatology area. Curative medicine and intensive care resources are prioritized in healthcare. Lack of knowledge or inexperienced on the topic may contribute to this situation. Several professionals that have difficulties in health communication should avoid it. Thus, implementing palliative care disciplines in the universities and health residency programs is the first step in improving care. Health education enables the qualification of professionals to deal with complex situations, such as diagnosing severe diseases and communicating about death, the lack of available therapy, and the reserved prognosis. It also enables greater acceptance of the patient to palliative care, since it improves the understanding and the accessibility to the information with quality when attended by trained professionals. ${ }^{7}$

A pregnant woman who is faced with fetal malformation deals with several kinds of griefs which begins from the moment of the discovery of the diagnosis. Griefing for a healthy baby that will not be born, griefing for a planned childbirth that will not take place, griefing over an idealized puerperium, and, many times, griefing for the loss of a child. Recognizing the pregnant woman and her family's necessity is essential in providing care. It is possible to apply palliative care, originally proposed for adults, adapted to perinatology. Thus, one should prioritize the comfort and relief of the patients' symptoms, communicate the evolution of the condition, respect the family's beliefs and religion, and have them participating actively in the therapeutic decisions, in welcoming grief in all its phases.

\section{References}

1. Gomes ALZ, Othero MB. Cuidados paliativos. Estud Av. 2016; 30 (88): 155-66.

2. WHO (World Health Organization). National cancer control programmes: policies and managerial guidelines. 2 ed. Geneva; 2002.

3. Munson D, Leuthner SR. Palliative care for the family carrying a fetus with a life-limiting diagnosis. Pediatr Clin North Am. 2007; 54 (5): 787-98.

4. Bolibio R, Jesus RCA, Oliveira FF, Gibelli MABC, Benute GRG, Gomes AL, Nascimento NBO, Barbosa TVA, Zugaib M, Francisco RPV, Bernardes LS. Cuidados paliativos em medicina fetal. Rev Med. 2018; 97 (2): 208-15.

5. Leuthner SR. Palliative care of the infant with lethal anomalies. Pediatr Clin North Am. 51. 2004; 51 (3): $747-59$.

6. Baile WF, Buckman R, Lenzi R, Glober G, Beale EA, Kudelka AP. SPIKES-A six-step protocol for delivering bad news: application to the patient with cancer. Oncologist. 2000; 5 (4): 302-11.

7. Li WW, Chhabra J, Singh S. Palliative care education and its effectiveness: a systematic review. Public Health. 2021; 194: 96108

Daniela Valle Almeida Figueredo 1

(iD https://orcid.org/0000-0002-2222-6858

Alex Sandro Rolland Souza 2

iD https://orcid.org/0000-0001-7039-2052

1,2 Instituto de Medicina Integral Prof. Fernando Figueira. Rua dos Coelhos, 300. Boa Vista. Recife, PE, Brazil. CEP: 50.070-902. 